\title{
JUGENDPSYCHIATRIE, FAMILIE UND ADOLESZENTE - SOZIALISATIONSTHEORETISCHE ANNÄHERUNGEN AN DIE FIGUR DES INSTITUTIONELLEN DRITTEN ALS STRUKTURELLEM KRISENAUSLÖSER
}

Julia Becher, Janina Schulmeister, Mirja Silkenbeumer

Goethe-Universität Frankfurt am Main, Fachbereich Erziehungswissenschaften, Institut für Sonderpädagogik

E-Mail: becher@em.uni-frankfurt.de

URL: https://www.uni-frankfurt.de/55945745/Julia_Becher_M_A

Goethe-Universität Frankfurt am Main, Fachbereich Erziehungswissenschaften, Institut für Sonderpädagogik

E-Mail: j.schulmeister@em.uni-frankfurt.de

URL: https://www.uni-frankfurt.de/55945808/Janina_Schulmeister_M_A

Goethe-Universität Frankfurt am Main, Fachbereich Erziehungswissenschaften, Institut für Sonderpädagogik

E-Mail: silkenbeumer@em.uni-frankfurt.de

URL: https://www.uni-frankfurt.de/55945511/Prof_Dr_Mirja_Silkenbeumer

Zitationsvorschlag:

Becher, Julia/Schulmeister, Janina/Silkenbeumer, Mirja (2021): Jugendpsychiatrie, Familie und Adoleszente - Sozialisationstheoretische Annäherungen an die Figur des institutionellen Dritten als strukturellem Krisenauslöser. In: Gesellschaft - Individuum Sozialisation (GISo). Zeitschrift für Sozialisationsforschung, 2 (2). DOI:

10.26043/GISo.2021.2.6

Link zum Artikel:

https://doi.org/10.26043/GISo.2021.2.6 


\title{
JUGENDPSYCHIATRIE, FAMILIE UND ADOLESZENTE - SOZIALISATIONSTHEORETISCHE ANNÄHERUNGEN AN DIE FIGUR DES INSTITUTIONELLEN DRITTEN ALS STRUKTURELLEM KRISENAUSLÖSER
}

\author{
Julia Becher, Janina Schulmeister und Mirja Silkenbeumer
}

\begin{abstract}
Werden Jugendliche aufgrund psychosozialer Krisen in einer jugendpsychiatrischen Klinik stationär behandelt, tritt diese als Repräsentantin des medizinisch-therapeutischen Handlungssystems und spezifische Form der institutionellen Krisenbearbeitung als institutioneller Dritter in die Dyade aus Adoleszenten und deren Familien ein. Gestützt auf Erkenntnisse rekonstruktiver Fallstudien wird der heuristische Mehrwert einer triadischen Perspektive auf Sozialisations- und adoleszente Bildungsprozesse im Schnittfeld der different strukturierten Sozialisationsräume Familie und Jugendpsychiatrie ausgeführt. Anhand zweier kontrastierender Fälle von psychiatrieerfahrenen Jugendlichen werden zwei Modi der familialen Bearbeitung der mit der Erweiterung des institutionellen Dritten verbundenen strukturellen Krisenkonstellation herausgearbeitet. Abschließend wird der Ertrag dieser triadische Strukturen berücksichtigenden Heuristik für eine fallrekonstruktive Forschungsperspektive auf Sozialisationserfahrungen in Institutionen diskutiert.
\end{abstract}

Keywords: Psychosoziale Krisen, Psychiatrie, Adoleszenz, Familie, Triade, Bildungsprozesse, Beziehungsdynamiken

\section{EINLEITUNG}

Im Rahmen dieses Beitrags stellen wir Erkenntnisse zu familialen Bearbeitungs- und Aushandlungsprozessen im Dreieck von Familie, Adoleszenten und Jugendpsychiatrie vor und beziehen uns dabei auf Ergebnisse unseres laufenden Forschungsprojekts "Transformations(an)forderungen im Übergang zwischen Familie, Kinder- und Jugendpsychiatrie und Psychotherapie und (Klinik-)Schule in der Adoleszenz". ${ }^{1}$ Vor dem Hintergrund einer triadischen Perspektive auf Sozialisationsprozesse in und zwischen Institutionen rücken wir die spezifische Dynamik ins Zentrum, die mit dem Hinzutritt der Kinder- und Jugendpsychiatrie als institutionellem Dritten sowohl im familialen Binnenraum als auch für die jeweiligen Akteur*innen der Familie entsteht. Dabei beleuchten wir die familialen Handlungsprobleme, die mit dem "Aufbruch“ der Familie nach außen durch die (temporäre) staatlich institutionelle und stellvertretend agierende Erziehungs- und Fürsorgeinstanz entstehen und die interaktiven und psychodynamischen Prozesse der Aushandlung, Positionierung, Abgrenzung und Differenzierung. Zentral zu berücksichtigen ist dabei die konstitutive Asymmetrie im Generationenverhältnis sowie die in der Lebensphase der Adoleszenz angestoßenen Entwicklungsprozesse, die sowohl auf eine ,innere“ Mobilisierung als auch auf von außen kommende Erwartungen zurückzuführen sind.

Nach einer kurzen Skizzierung einiger wichtiger Studien in dem Feld setzen wir uns in einem ersten Schritt aus einer sozialisations- und interaktionstheoretischen Perspektive mit der Figur des Dritten im familialen und um die Klinik erweiterten sozialen Gefüge auseinander und fragen

\footnotetext{
${ }^{1}$ In dem Projekt fokussieren wir Erfahrungen von Jugendlichen, die stationär in einer Kinder- und Jugendpsychiatrie behandelt werden. Insbesondere das Erleben des Übergangs vom familialen Raum und der Stammschule in die Klinik(schule) steht im Zentrum des Projekts (vgl. Silkenbeumer et al. 2020; Becher/Schulmeister 2020; Hornung/Becher 2019).
} 
nach der Umgestaltung von familialen Beziehungsdynamiken, die die Jugendpsychiatrie potenziell anzustoßen vermag (2). Nach einer kurzen Erläuterung unseres methodischen Vorgehens (3) zeigen wir darauffolgend entlang zweier kontrastierender Fälle durch den Klinikaufenthalt ausgelöste Veränderungen von Beziehungsdynamiken zwischen psychiatrisch behandelten Adoleszenten und ihren Eltern (4), die in engem Zusammenhang stehen mit der Rolle und Bedeutung, die dem institutionellen Dritten im intergenerationalen Beziehungsgeflecht zugeschrieben wird. Abschließend (5) diskutieren wir das erkenntnistheoretische Potential dieser um den Dritten erweiterten Konzeption für das Feld der rekonstruktiven Sozialisations- und Adoleszenzforschung.

\section{SOZIALISATIONSTHEORETISCHE PER- SPEKTIVEN AUF DIE BEDEUTUNG DES INSTI- TUTIONELLEN DRITTEN}

Forschung, in der nach dem sozialisatorischen Gehalt der strukturellen Erweiterung und Umgestaltung bestehender familialer Beziehungsgeflechte durch einen institutionellen Dritten - vor allem aus einer subjekttheoretischen Perspektive - gefragt wird, stellt ein markantes Desiderat dar. Studien zu Normierung und Medizinisierung von Kindheit und Jugend, die nach der Bedeutung des medizinisch-therapeutischen Handlungssystems fragen, haben Haubl und Liebsch (2010) vorgelegt. Aus soziologischer und sozialpsychologischer Perspektive gehen sie der Frage nach, wie Jungen zwischen sieben und 14 Jahren die innen attestierte psychiatrische Diagnose einer $A D(H) S$ und deren psychopharmakologische Behandlung verstehen, und stellen Prozesse der „Entgrenzung von Erziehung und Medizin“ im Zuge des Einsickerns medizinischer Deutungen in den familialen Raum fest (Liebsch et al. 2013). Im Kontext von Kindervorsorgeuntersuchungen fokussieren Bollig et al. (2012) das Verhältnis von Familie und Medizin und arbeiten die medizinische Deutungshoheit im Arbeitsbündnis zwischen Ärzt*innen und Eltern heraus, die sich über das Kinderuntersuchungsheft als Erziehungsfragment herstellt und Zuständigkeiten für den kindlichen Körper erzeugt. Die Konstellation aus Institution bzw. Organisation, Professionelle*r, Familie und Patient*innen nimmt Peter (2006) anhand der medizinischen Versorgung in Kliniken aus einer familien-, professions- und organisationssoziologischen Perspektive in den Blick und rekonstruiert Bedingungen der Herstellung und Strukturierung von Kooperationsverhältnissen. Mit der Studie "Störer und Gestörte" (von Freyberg/Wolff 2005) werden (sozial-)pädagogische und schulische Kontexte fokussiert und es sind die biografischen Verläufe "nicht-beschulbarer" Jugendlicher und die sie und ihre Familien begleitenden, immer härter erfolgenden institutionellen Weichenstellungen, die aus soziologischer und psychoanalytischer Perspektive rekonstruiert werden. Das Spannungsverhältnis von Familie und Professionellen bei staatlich verordneten Eingriffen in die Familie - z. B. bei Kindeswohlgefährdung - wird aus sozialpädagogischer Perspektive bei Bütow et al. (2014) untersucht und Gehres und Hildenbrand (2008) gehen in ihrer Studie der sozialisatorischen Bedeutung des Dreiecks bestehend aus Herkunfts-, Pflegefamilie und Jugendamt nach und arbeiten deren Bedeutung für kindliche und adoleszente Identitätsbildungsprozesse heraus.

Sozialisationsprozesse im Kontext von Dreieckskonstellationen bzw. der „Figur des Dritten“2 (Eßlinger et al. 2010) zu betrachten, bedeutet im Sinne des vorliegenden Beitrags, die Erfahrungen psychiatrieerfahrener Adoleszenter ihrer triadischen Struktur nach zu entflechten und auf ihre damit für familiale Aushandlungs- und adoleszente Bildungsprozesse relevanten Dynamiken hin zu befragen. Wenn man den Hinzutritt der psychiatrischen Institution aus der Perspektive eigenlogisch strukturierter familialer bzw. generationaler Dynamiken betrachtet, in dem adoleszente Entwicklungsprozesse mit familialen und externen Erwartungen und Normalitätsanforderungen in Konflikt geraten können, scheint die Figur des Dritten gewinnbringend.

\footnotetext{
2 Triadische Konstellationen werden in unterschiedlichen wissenschaftlichen Disziplinen und Schulen diskutiert. So findet sich die Triade sowohl als Beziehungsfigur familiendynamischer Prozesse, wie in der strukturtheoretischen ( $v$. a. Allert 1998; Oevermann 2001) und psychoanalytischen und systemischen Familientheorie (z. B. Fivaz-Depeursinge/Corboz-Warnery 2001), als auch in kulturwissenschaftlichen (z. B. Eßlinger et al. 2010) und sozialtheoretischen (z. B. Fischer 2008; Simmel 1992) Perspektiven.
} 
Denn wenn wir die familialen, intern durchaus differierenden Erwartungen an die Psychiatrie ins Zentrum stellen, können hierdurch Konflikte in den Blick kommen, für deren Prozessierung die Positionierung des Dritten durch die anderen „Parteien“ mitentscheidend wird. Nach Simmel kann dem hinzukommenden Dritten sowohl trennende als auch verbindende Kraft zukommen, in jedem Fall tritt mit inm eine strukturelle Störung und Dynamisierung der zuvor dyadischen Beziehung ein (Simmel 1992, 114 f.). Eine Triade lässt sich in drei Dyaden ausdifferenzieren, deren Positionen in unterschiedlichem Maße miteinander in Verbindung stehen oder aber auf Distanzierung und Trennung verweisen können. Einer „symbiotisch-dyadische[n] Abschließung und Verschmelzung" steht "ein Schritt zu zentrifugaler, triangulierender Öffnung" (Grieser 2017. 25) gegenüber.

Diesen Dynamiken des Schließens und Öffnens, des Changierens zwischen Nähe und Distanz, kommt in der Adoleszenz eine herausgehobene sozialisatorische Bedeutung zu. Als potentielles Moratorium der Um- und Neustrukturierung und „psychosozialer Möglichkeitsraum“ (King 2013, 39) wird Adoleszenz insbesondere dort bedeutsam, wo Prozesse der Individuierung und Dimensionen von Transformation in den Blick geraten; dies auch unter Berücksichtigung der in generative Sorge- und Erziehungsverhältnisse strukturell eingeschriebenen Machtasymmetrien, damit verbundenen Verletzlichkeiten und unterschiedlichen Chancenstrukturen (King 2015; Janssen 2018).

Findet sich im Konstellationsgefüge der Familie ${ }^{3}$ in der Regel eine hohe Dynamik im Wechsel möglicher Positionen (Allert 1998), besitzt eine gesellschaftliche Institution eine vielfach größere Statik und zugleich affektive Neutralität. Mit Blick auf das Verhältnis von Familie, Institution und Profession wird die Differenz zwischen diffus strukturierten Familienbeziehungen, formal-spezifischen Rollenbeziehungen in der Klinik und dem sich sowohl aus diffusen als auch spezifischen Beziehungsanteilen zusammensetzenden professionellen Arbeitsbündnis bedeutsam (Oevermann 1996). ${ }^{4}$ Dies kommt besonders dort zum Tragen, wo sich die Frage nach Anerkennungs- und Aneignungsmöglichkeiten externer Deutungs- und Interventionsangebote des Dritten durch die Familie als soziales System mit je unterschiedlich durchlässigen Binnen- und AuBengrenzen stellt (Stierlin et al. 1980). Die Institution der Psychiatrie lässt sich dabei durch die Brille der von Simmel herausgearbeiteten Figur des Unparteiischen und des Vermittlers betrachten (Simmel 1992, 125 ff.), der durch die Familie als „Vermittlungsinstanz" zwischen Privatheit und Öffentlichkeit allererst einbezogen wird:

Der hinzukommende Dritte stört nämlich nicht nur das reziproke Wechselspiel innerhalb der Dyade, sondern bringt damit auch ein Element von Indirektheit, Distanzierung und Fremdbeobachtung ins Spiel, das den Nukleus jener Überpersönlichkeit und Objektivation bildet, mit dem institutionelle Einheiten „dem Einzelnen gegenübertreten“. (Koschorke 2010, 51)

Der Dritte kann so Perspektivenübernahmen ermöglichen und die Akteur*innen dazu bringen, sich im Rahmen einer Ordnung zu verständigen, die über den subjektiven und affektiv besetzten Standpunkt hinausgeht. Zugleich können in den Dritten aber auch Erwartungen projiziert werden, die über den Auftrag der Institution hinausgehen. Die Jugendpsychiatrie kann als „auf Dauer gestellte und zum abstrakten Prinzip erhobene Figur [...] des Dritten“ (ebd., 53) betrachtet werden, die der Krise in der Familie auf eine ganz bestimmte, ihrem inhärenten Deutungssystem entsprechenden Form begegnet. Dabei sind die Interventionsformen der jugendpsychiatrischen Profession mit ihren spezifischen Behandlungstechniken zu unterscheiden von den Dynamiken, die zwischen den prozessierenden Akteur*innen entstehen und fernab geplanter therapeutischer und erzieherischer Interventionen ablaufen und dabei sozialisatorische Prozesse initiieren.

In dem von uns untersuchten Phänomen wird die Familie durch den Hinzutritt des institutionellen

\footnotetext{
${ }^{3}$ Zur Unterscheidung der „äußere[n] und innere[n] Bedeutung der Triade“ und einer damit verbundenen Auseinandersetzung mit der Kritik am Modell der familialen Triade siehe King (2018).

${ }^{4}$ Auf die notwendige Differenzierung des spannungsreichen Bedingungsgefüges von Profession und Institution weist Peter (2006, 70 f.) am Beispiel von Krankenhäusern hin.
} 
Dritten somit nicht nur auf der Ebene konkreter Akteur*innen erweitert. Auch gewinnen durch diese Erweiterung die unterschiedlichen Beziehungskonstellationen und -dynamiken eine andere Qualität und werden damit potentiell umgestaltet. In diesem um die Jugendpsychiatrie erweiterten Dreieck kommt Dezentrierung strukturell in dreifacher Weise eine herausgehobene Bedeutung: zum einen auf der „Ebene der Realbeziehungen“" (Grieser 2017, 24), wenn durch den Hinzutritt der Klinik der Familie sowie den Jugendlichen eine außenstehende und vor dem Hintergrund familialer Dynamiken weitgehend unbefangene Perspektive gegenübergestellt wird: ${ }^{5}$ zum zweiten auf der Ebene der „inneren Welt" (ebd.), als Möglichkeit zur Perspektivenübernahme und Einnahme einer beobachtenden Position über dyadische Beziehungskonstellationen hinaus - entsprechend eines Wechsels von einer „Unmittelbarkeit von selbstbezogener Erfahrung und damit einhergehendem Affekt in eine sich selbst betrachtende reflexive Position" (King 2013, 129). Während diese beiden Aspekte für alle triadischen Konstellationen gelten, zielt die dritte Ebene auf eine für die Jugendpsychiatrie spezifische Dimension, nach der Dezentrierung ein maßgebliches Ziel therapeutischen Handelns darstellt (vgl. Grieser 2017). Die medizinischen Deutungsansprüche devianten Verhaltens als "Krankheit" dringen - durch (Kinder) Ärzt*innen oder ambulante Psychotherapeut*innen, häufig schon vor Einbezug der Klinik - in den binnenfamilialen Raum ein und sind verknüpft mit Normalitätsvorstellungen und Transformationsanforderungen, die eine große Nähe zu Fragen der Erziehung aufweisen können. Diese stellvertretenden Deutungen und $\mathrm{Zu}$ schreibungen des jugendpsychiatrischen Handlungssystems können für die Adoleszenten und Familien mit Herausforderungen der (Neu-)Positionierung, Abgrenzung, Aushandlung und damit der (potentiellen) Umgestaltung familialer Beziehungen verbunden sein.

Dass die Situation von Eltern, deren adoleszente Kinder stationär psychiatrisch behandelt werden, als Krise ${ }^{6}$ betrachtet werden kann, wird deutlich, wenn man sich vergegenwärtigt, dass die räumliche Trennung des Kindes von der Familie maßgeblicher Teil der Behandlung ist; bei einer stationären und vor allem geschlossenen Unterbringung besteht zuweilen für einige Wochen kein Kontakt. Streicht man hier die Binnenperspektive der Adoleszenten stärker heraus, ist es auch die temporäre Trennung von den Peers, die es zu "organisieren“ und auszuhalten gilt. Diese Separation selbst als Teil des Heilungsprozesses zu erfahren, kann auf der Ebene der Eltern auch den Eindruck evozieren, als primäre Bezugspersonen „versagt“ zu haben. Neben der Trennung vom primären Sorgemilieu können Schuldgefühle schon dadurch entstehen, dass der Klinikaufenthalt mit dem temporären Übertrag von (Erziehungs-)Verantwortlichkeiten der Familie einhergeht.

Diese Überlegungen zur Erweiterung und strukturellen Umgestaltung der familialen Triade durch die Jugendpsychiatrie lassen sich, sensibilisiert durch das Buchholzsche Modell der institutionellen Triade (Buchholz 1993, 193-196), analytisch weiter auffächern und auf ihren sozialisatorischen Gehalt hin untersuchen. Zwar ausgehend vom familientherapeutischen Kontext skizziert, doch nicht weniger fruchtbar für eine sozialisationstheoretische Betrachtung des Phänomens nimmt Buchholz die in der Triade Therapeut*in - Familie - Patient*in möglichen (idealtypischen) Koalitionen und Dyadenspaltungen in den Blick, wie sie im Allgemeinen für Dreieckskonstellationen bedeutsam sind. Bei „einer solchen Spaltung können erhebliche dynamische Kräfte freiwerden, die ihre Wirksamkeit in einem sich selbstbestätigenden Muster von Täter-Op-

\footnotetext{
${ }^{5}$ Wie unbefangen diese dritte Perspektive wahrgenommen wird, hängt nicht unwesentlich davon ab, wie der Eintritt in die Klinik prozessiert wird. Mit zunehmendem Grad der Verfestigung der Beziehung zwischen professionellen Akteur*innen und Klient*innen wird die psychodynamische Involvierung in den Behandlungsprozess zunehmend relevant. Die mit der Neutralität verbundenen Anforderungen an den Dritten beschäftigen auch Stierlin (2001, 237 ff.), der die Variationsformen therapeutischer Parteinahme herausarbeitet, die im Zuge dessen Prozesse initiieren können, die therapeutischen Zielen entgegenlaufen.

${ }^{6}$ Zur Krise als zentralem Begriff einer strukturtheoretischen Sozialisations- und Bildungstheorie vgl. Oevermann (2004).
} 
fer- [bzw. Retter-Zuschauer; Anm. d. Verf.] Konstellationen entfalten“" (ebd., 194). Er unterscheidet drei Spaltungskonstellationen, in denen sich jeweils zwei Akteur*innen zusammenschließen und der Dritte aus dem Bündnis ausgeschlossen wird. Während in der ersten Konstellation die Allianz zwischen Patient*in und Therapeut*in unter Ausschluss der Angehörigen zentral wird, ist mit der zweiten Konstellation auf die Dyade zwischen Angehörigen und Therapeut*in unter Ausschluss der uneinsichtigen Patient*innen abgehoben - eine Konstellation, auf die Goffman (2014) mit Blick auf zwangseingewiesene Patient*innen in der Psychiatrie abhebt. Konstellation drei rückt den Zusammenschluss von Angehörigen und Patient*in ins Zentrum, durch den die Therapeut*innen ausgeschlossen werden.

Während die tripolaren Stellungen dieses Modells ${ }^{7}$ durch die konkreten Akteur*innen Familie - Adoleszente - Klinik (bzw. Behandelnde) problemlos eingenommen werden können, machen die eingangs aufgeführten sozialisations- und adoleszenztheoretischen Überlegungen eine Konkretion dieses Modells notwendig, das uns schließlich für sozialwissenschaftliche rekonstruktive Forschung zu Institutionenerfahrungen psychiatrieerfahrener Adoleszenter und deren Familien als fruchtbar erscheint. Denn adoleszente Individuation verweist per se auf generative Andere, auf Prozesse der zweiseitigen Ablösung und auf eine allmähliche Verselbstständigung im Prozess der Sozialisation, die die Entwicklung hin zu relativer Autonomie ermöglicht. Somit werden auch Abhängigkeits- und Sorgeverhältnisse berührt, Erfahrungen von Macht und Ohnmacht sowie von Anerkennung und Missachtung.
Anhand einer Fallkontrastierung zweier Jugendlicher unseres Projekts beleuchten wir im Folgenden die durch die strukturelle Erweiterung der Dyade aus Eltern und Adoleszenten durch die Klinik angestoßenen familialen Aushandlungen und Konfliktdynamiken.

\section{METHODISCHER ZUGANG IM QUALITATI- VEN LÄNGSSCHNITT}

Im Rahmen unseres Projekts führten wir zu vier unterschiedlichen Zeitpunkten insgesamt 24 bildungsbiografische Interviews und Familiengespräche mit bislang sieben Minderjährigen, die stationär kinder- und jugendpsychiatrisch behandelt wurden. Die ersten beiden Gespräche wurden kurz nach dem Klinikaufenthalt oder kurz vor der Entlassung geführt und die nächsten beiden Einzel- und Familiengespräche nach weiteren vier bis zwölf Monaten. ${ }^{8}$ Methodologisch ist das Projekt im Bezugsrahmen sinnrekonstruktiver Forschungsverfahren verortet - und hier im Spezifischen in der objektiven Hermeneutik (Oevermann 2000) -, um das Zusammenspiel von latenten und manifesten Sinndimensionen sequenzanalytisch aufschließen zu können. Die folgende ergebnisorientierte Darstellung basiert auf objektiv-hermeneutischen Fallrekonstruktionen die zu Einzelfallstudien verdichtet wurden; ${ }^{9}$ diese können an dieser Stelle nicht in ihrer Komplexität abgebildet werden. Im Kern richtet sich die Analyse auf die sozial- und sinnstrukturierte Prozessierung des Klinikaufenthalts, die subjektiven und biografischen Aneignungsprozesse und damit verbundene intergenerational strukturierte Aushandlungen. Dabei dient die Erhebung

\footnotetext{
${ }^{7}$ Dabei muss darauf hingewiesen werden, dass dieses Triadenmodell grob vereinfacht ist und es sich immer um eine Vielzahl paralleler Dreieckskonstellationen handelt, die neben den Behandelnden auch weitere medizinische und pädagogische Fachkräfte und die Gruppe der Gleichaltrigen umfassen kann (Buchholz 1993, 193).

${ }^{8}$ In einigen Fällen ergaben sich Abweichungen von diesem Vorgehen: zum Teil aufgrund der fehlenden Möglichkeit, zu den vorgesehenen vier Zeitpunkten Interviews führen zu können, und zum Teil, um weitere biografische Statuspassagen in den Blick zu bekommen. So liegen uns im Fall Sebastian neben zwei Einzel- und zwei Familiengesprächen außerdem zwei Elterngespräche vor, die zwei Jahre nach dem Klinikaufenthalt und kurz nach seinem Schulabschluss bzw. nach Einstieg ins Berufsleben geführt wurden. Die letzten beiden Elterngespräche werden in diesem Beitrag nicht berücksichtigt, da sie einen anderen thematischen Schwerpunkt aufweisen. Im Fall Olivia führten wir in zwei Jahren insgesamt vier Gespräche (zwei Einzelgespräche, ein Gespräch mit der Mutter, ein Familiengespräch). In diesem Fall konnten wir das erste Gespräch mit Olivia vier Wochen vor ihrem Klinikaufenthalt führen.

${ }^{9}$ Zum methodischen Vorgehen siehe Wernet (2009).
} 
im qualitativen Längsschnitt ${ }^{10}$ der Rekonstruktion individueller adoleszenter und familialer Bildungs-, $\quad$ Entwicklungs- und Transformationsprozesse im Kontext von als Krankheit bezeichneten psychosozialen Krisen und sozialisatorischen Erfahrungen in der Institution der Kinder- und Jugendpsychiatrie. Zentral für eine qualitative Längsschnittperspektive ist das „Spannungsfeld von Transformations- und Reproduktionsprozessen in ihrer kontext- und zeitspezifischen Dimensionalität" (Thiersch 2020, 16). Diese eröffnet einen „methodischen Zugang zur Gegenüberstellung und Kontrastierung der Deutungen, Praktiken und Bewertungen zu unterschiedlichen Zeitpunkten und damit Möglichkeiten, die Veränderungen in der Wahrnehmung dieser Passagen und Pfade sichtbar zu machen" (ebd.).

Die Erhebung unterschiedlicher Gesprächskonstellationen und damit auch unterschiedlicher Interaktionen und Akteursperspektiven soll zum einen Aufschluss über das konkrete familiale Adressierungs- und Aushandlungsgeschehen geben und zum anderen die Entwürfe der Jugendlichen selbst und ihre Auseinandersetzung mit institutionellen und familialen Erwartungen der Analyse zugänglich machen.

\section{SEBASTIANUND OLIVIA - ZWEI KON- TRASTIERENDE DYNAMIKEN HINSICHTLICH DER BEDEUTUNG DES INSTITUTIONELLEN DRITTEN FÜR FAMILIALE BEARBEITUNGS- UND AUSHANDLUNGSPROZESSE}

Bei den folgenden Fällen, die wir Sebastian und Olivia nennen, handelt es sich um zwei maximal kontrastive Fälle unseres Samples hinsichtlich der familialen Bearbeitungsmodi der strukturell angestoßenen Krisenkonstellation. Zentral bei diesen Fällen ist die familiale Prozessierung der Klinikeinweisungen, anhand derer die strukturell notwendig werdenden Entscheidungs- und Aushandlungsprozesse in ihrer Bedeutung auf Beziehungsdynamiken im institutionellen Dreieck prägnant verdeutlicht werden können.

\subsection{Sebastian-Ambivalente Delegationen fami- lialer Erziehungskrisen an den institutionellen Dritten}

Sebastian ist zum Zeitpunkt des ersten Interviews 14 Jahre alt und das jüngste von drei Kindern. Nach der Grundschule wird er auf ein Gymnasium versetzt, wiederholt die fünfte Klasse und wechselt aufgrund mangelhafter Noten in der sechsten Klasse in eine Realschule. Sebastian beginnt ab diesem Zeitpunkt unregelmäßig die Schule zu besuchen, bis er ihr schließlich ganz fernbleibt und sich stattdessen größtenteils zu Hause oder bei Freund*innen aufhält. Sebastians Eltern, die sich nicht mehr im Stande dazu sehen, den Schulbesuch und die Einhaltung der Schulpflicht ihres Sohnes sicherzustellen, veranlassen schließlich durch eine familiengerichtliche Genehmigung zur freiheitsentziehenden Unterbringung und einer polizeilich begleiteten Zuführung in die Institution den vollstationären Aufenthalt in einer jugendpsychiatrischen Klinik. ${ }^{11}$ Nach knapp zwei Monaten wird Sebastian aufgrund eines Verstoßes gegen die Klinikvorschriften für einen Monat der Klinik verwiesen - in dieser Zeit besucht er weiterhin die Klinikschule. Sein zweiter Aufenthalt im Anschluss an den Verweis dauert erneut etwa acht Wochen, bis er geplant entlassen wird. Das objektive Datum der Zwangseinweisung stellt sich uns in Sebastians Fall als primärer Dreh- und Angelpunkt für die Frage nach in der institutionellen Triade wirksam werdenden Beziehungs-, Ablösungs- und Individuierungsdynamiken und als zentrales Krisenereignis dar.

\footnotetext{
${ }^{10}$ Zu methodischen und methodologischen Überlegungen zur Längsschnittperspektive in Verbindung mit sozialisations- und adoleszenztheoretischen Fragestellungen siehe Becher/Schulmeister (2020). Zu rekonstruktionsmethodologischen Problemstellungen hinsichtlich der Analyse des "Gewordenseins“ und zum Spannungsverhältnis von Rekonstruktion und Transformation aus der Perspektive der objektiven Hermeneutik siehe Wernet (2020).

${ }_{11}$ Psychiatrische Einweisungen gegen den Willen der Betroffenen werden i. d. R. über eine faktische (oder drohende) Selbst- und/oder Fremdgefährdung und den Ausschluss alternativer Interventionen - bspw. (sozial-)pädagogische Einflussnahmen - begründet. Während anhaltendes schulverweigerndes Verhalten juristisch als eine Form chronischer Eigengefährdung gedeutet und als Begründung für einen psychiatrischen Aufenthalt in Anschlag gebracht werden kann (Rüth 2011, 556), drängt sich zudem die Frage nach dem Einfluss erzieherischer Absichten auf, wie sie sich in der Rekonstruktion bei Sebastian durchaus zeigen.
} 
Für die Einweisung finden sich in den Gesprächen unterschiedliche Deutungen, die wir zum einen durch einen exemplarischen Auszug aus dem ersten Einzelgespräch mit Sebastian und, für die Perspektive der Eltern, aus dem ersten Familiengespräch hier aufgreifen möchten:

Sebastian: Also in die Klinik rein isch war ja erst mal in den Ferien da es war am Anfang schwierisch weil isch halt nisch wollte aber isch war ja mitm Beschluss da [...] (I1: Was heißt mit nem Beschluss da?) äh isch wollt nämlisch nisch hin und dann ham meine Eltern beim Rischter $n$ rischterlichen Beschluss eingeklagt dass isch da hin muss dann hab isch halt nachgegeben und bin dann halt auch mit meinen Eltern hingefahrn und mit der Polizei.

Vater: [...] dass er da [in die Schule; Anm. d. Verf.] net hingegangen is das ging dann immer mit Übelkeit Erbreschen einher dann iss er hingegangen dann hats nach zwei Stunden ja holn se ihren Sohn ab und das hat sich ja dann unheimlisch hochgeschaukelt woraufhin ma dann zum Arzt is um da einfach medizinisches Problem auszuschließen joa hat auch das dann wieder verweigert (Mutter: ja) und letzendlich simma dann zum Amtsgericht un ham dann uns ne Verfügung geben lassen dass mer ihn zwangseinweisen können und das ham wa dann halt auch gemacht [...].

Die Dreieckskonstellation aus Sebastian, seinen Eltern und der Klinik wird durch die Entscheidung seiner Eltern als Initiator*innen der erzwungenen Einweisung, vermittelt über die Genehmigung seitens des Familiengerichts, allererst errichtet. Hier stoßen wir auf eine triadische Konstellation, die sich im Wesentlichen erst durch die starke Betonung der asymmetrischen Beziehung zwischen Eltern und ihrem minderjährigen Kind in bestimmter Weise konstelliert. Auf die Frage, wie Sebastian den Klinikeintritt erlebt hat, führt er aus, dass er gegen seinen Willen mit einem „Beschluss" - als offizielles, amtliches und somit auch machtvolles Dokument - durch Dritte eingewiesen und somit über ihn als Rechtssubjekt verfügt wurde. Neben dem Richter, als Repräsentant der Judikative und Staatsgewalt, benennt er konkret seine Eltern, die den Beschluss zur Zwangseinweisung „eingeklagt“ und somit in hochgradig formalisierter Form ihr Recht als
Sorge- und Erziehungsberechtigte in Anschlag gebracht und durchgesetzt haben. In der elterlichen Delegation des im familialen und schulischen Kontext ausgebrochenen und dort nicht länger als bearbeitbar betrachteten Erziehungsproblems - Sebastian kommt den an inn gestellten Erwartungen nicht nach, was schließlich in einem unlösbaren Familienkonflikt gipfelt - an das medizinisch-therapeutische Handlungssystem in Gestalt der Jugendpsychiatrie kommt einerseits die maximale Machtdemonstration Sebastians Eltern zum Ausdruck, anderseits aber auch die eigene Ohnmacht und Sorge um das Wohlergehen des eigenen Kindes, dessen hohe schulische Fehlzeiten ein mögliches, vielleicht auch gravierendes Teilhabedefizit zur Folge haben könnten. Mit dieser Delegation der im Kern auf eine familiale Erziehungskrise verweisenden Dynamik an die Klinik, die für die Zeit der stationären Behandlung auch eine (temporäre) Übertragung des Erziehungs- und Sorgeauftrags mit sich bringt, wird der Zweifel an Sebastians Entscheidungsfähigkeit in der institutionellen Dreieckskonstellation offengelegt und betont, so auch die Einschränkung dessen Selbstbestimmung durch die Eltern. Sebastian wird als Adoleszenter hier auf die Position des von Erwachsenen abhängigen Kindes zurückgeworfen, dem eigenmächtige Verantwortlichkeiten und der Status eines entscheidungsfähigen Subjekts abgesprochen werden. Denn trotz seines Widerwillens und seiner klaren Entscheidung, nicht in die Klinik gehen zu wollen, muss er schließlich aufgrund des gerichtlichen Beschlusses in die Institution eintreten; es bleibt inm in dieser polizeilich kontrollierten Situation nichts anderes übrig, seine geschilderte Nachgiebigkeit ist alternativlos. Im Narrativ des Vaters wird Sebastians leiblicher Ausdruck des Erbrechens in erster Linie als Widerstand gegen seine Eltern gedeutet, dem man in letzter Instanz auch mit staatlicher Gewalt zu begegnen hat. Die medizinische Institution soll dabei ebenso wie der Rechtsstaat als verlängerter Arm der elterlichen Erziehungspraxis dienen, um so den scheinbar kontinuierlichen Widerstand des opponierenden Sohnes aufzuheben.

Konfrontieren wir diese Überlegungen zur Struktur der Dreieckskonstellation in Sebastians Fall nun mit der Frage nach darin potenziell verbor- 
genen Implikationen für adoleszente Individuierungsprozesse und schließlich familiale Beziehungsdynamiken, erscheinen vor allem zwei Dimensionen gewichtig: zum einen die bereits angesprochene massive Einschränkung Sebastians Handlungsautonomie und dessen „Statusverlust" als Adoleszenter, der im Rahmen generativer Machtverhältnisse mit der Positionierung als von den Eltern und Therapeut*innen abhängiges Kind zu Tage tritt; zum anderen die mit der erzwungenen Einweisung verbundene, von außen induzierte Trennung von der Familie und dem gewohnten sozialen Umfeld, für die er sich nicht eigenmächtig - bspw. im Sinne eines heilsamen Rückzugs, wie es bei Olivia der Fall sein wird - entscheidet, sondern als unausweichlicher "brute fact“ physisch wie psychisch über inn hereinbricht. Sensibilisiert durch das Buchholzsche Modell der institutionellen Triade ließe sich von einer Koalition zwischen Eltern und Klinik sprechen, durch die Sebastian von seinen Eltern und der Klinik ausgeschlossen wird. Die in der Triade enthaltene und auf therapeutische Prozesse ausgerichtete Zweierbeziehung zwischen der Klinik und Sebastian wird durch den Umstand der Zwangseinweisung und Sebastians Widerwillen strukturell erschwert, auch wenn an dieser Stelle eine (nachträgliche) Aneignung noch nicht ausgeschlossen werden kann. Dominantes Familiennarrativ zur Entlassung und dem Klinikaufenthalt ist dann jedoch die gemeinsame Ablehnung der Klinik, insbesondere der psychiatrischen Behandlung. Dazu ziehen wir exemplarisch eine Aussage des Vaters heran: „Vater: [...] ich hat das Gefühl die wollen den da nit mehr haben (Sebastian: ja) so kam das für mich rüber."

Die Dyaden zwischen Klinik und Eltern und Sebastian und seinen Eltern erfahren eine Umdeutung. Trotz der verschiedenen Narrative über die erzwungene Einweisung in die Psychiatrie teilt sich die Familie letztlich eine gemeinsame Erzählung im verteidigenden Bündnis gegen die Klinik. In der geteilten „Opferrolle“ ist die Familie als Kollektiv von der Klinik hintergangen und enttäuscht worden. Ein erster binnenfamilialer Zusammenhalt wird dadurch (wieder)hergestellt, dass die potentiell die Familie selbst in Frage stellende psychiatrische Diagnose und Behandlung als ungültig - und nicht als Möglichkeit einer Dezentrierung - illegitimiert wird; die Übersetzung der medizinischen Deutungsbestände in die Familie gelingt nicht und der durch den Hinzutritt der Jugendpsychiatrie erweiterte Aushandlungsbereich der psychosozialen Krise wird ungelöst zurück in den familialen Raum verschoben. Im Fall der jugendpsychiatrischen Behandlung haben wir es demnach mit widerstreitenden Deutungen zu tun. Aufgrund der intergenerational gezogenen Machtasymmetrie werden diese zwar deutlich von den Erwachsenen dominiert, jedoch geraten diese im Zuge adoleszenter Ablösungsbewegungen und damit wechselnder Kräfteverhältnisse verstärkt unter Druck, was sich in der Harmonisierung einer feindlich nach außen hin gerichteten Perspektive Ausdruck verschafft. Der Ausschluss des psychiatrischen Dritten restabilisiert die Familie zwar hinsichtlich der Krise, die mit der Zwangseinweisung verbunden war, aber belässt die Frage nach den innerfamilialen Verbindlichkeiten jenseits der Abgrenzung von der medizinischen Institution letztlich ungeklärt.

\subsection{Olivia - Der institutionelle Dritte in seiner Funktion als Bündnispartner für adoleszente $\mathrm{Ab}$ - lösungsprozesse}

Olivia ist zum Zeitpunkt des ersten Interviews 16 Jahre alt und das jüngste dreier Kinder. Ihre Eltern trennen sich kurz nach ihrer Einschulung und sie lebt seitdem zusammen mit ihrer Mutter. Zu ihrem Vater bestand nur unregelmäßig Kontakt und wurde inzwischen gänzlich abgebrochen. Ihre Kindheit ist von vielen Umzügen geprägt sowie von der schweren Erkrankung ihres ältesten Bruders. Als Olivia 15 Jahre alt ist, zieht sie mit ihrer Mutter zu deren neuen Freund, sodass sie die Schule wechseln und ihre feste Freundin und Peers hinter sich lassen muss. Kein Jahr später entscheidet sich Olivia aufgrund von Depressionen und dem dadurch verhinderten Schulbesuch dazu, eine stationäre psychiatrische Behandlung zu beginnen. Der freiwillige Eintritt in eine psychiatrische Klinik stellt den entscheidenden Unterschied zu Sebastian dar, sodass dieser Fall als Kontrastfall gesehen werden kann, in dem die triadischen Dynamiken flexibler und die Möglichkeiten der Dyadenbildung anders gelagert sind. Wir finden hier einen Aneignungsprozess, der die Klinik in ihrer Funktion als ablösenden Dritten in den Mittelpunkt rückt, der adoleszente Dynamiken in Gang setzt und stabilisiert. Wenngleich 
die psychodynamischen Prozesse zwischen Mutter und Tochter ungleich komplexer sind, tritt Olivias Mutter im Gegensatz zu der gravierenden Infragestellung von Autonomie im Fall Sebastian eher in einer Funktion des unterstützenden Beratens auf. Dadurch, dass Olivia die an sie herangetragene Krisendeutung der Klinik in ihren Selbstentwurf integriert, sich die Adressierung als krank und behandlungsbedürftig zu eigen macht - jedoch keinesfalls im Sinne einer Zäsur bereits vorhandener Selbstentwürfe, sondern als deren Fortsetzung -, eignet sie sich die Perspektive der Klinik in einer für sie dezentrierenden Weise an. Exemplarisch wird die spezifische Erfahrung des bevorstehenden Klinikaufenthalts an folgender Stelle deutlich, die dem zweiten Einzelgespräch mit Olivia entnommen ist und auf die Frage folgte, wie es ihr seit dem letzten Gespräch - welches einen Monat vor ihrem Klinikaufenthalt stattfand - ergangen ist. Auch in diesem Fall zeigen sich Ambivalenzen gegenüber der Klinik, die aber im Vergleich zu der oben bei Sebastian herausgearbeiteten Konstellation anders gewichtet werden müssen:

Olivia: Also: als ich des: des war irgendwie voll chaotisch weil halt (.) ähm (.) also meine Mutter hat sich natürlich über den Platz gefreut weim lange gewartet haben und dann: als ich halt (.) von der Schule nach Haus gekommen bin hatte son paar Kumpels von: ä Schule dabei und (.) wollten eigentlich so (.) ganz normal chillen hier und dann: sagt meine Mutter in der öffentu ja du hast einen Platz gekriegt (.) nächste Woche ich so (.) $\ddot{a}$ (.) weil ich zu meiner damaligen Freundin fahren wollte (.) u: ich so des is doof(.) weil genau dann ich ${ }^{\circ}$ da fahrn wollte ${ }^{\circ}$ (lachend)

Im Kontrast zu Sebastian beginnt Olivia ihre Erzählung nicht in der Klinik, sondern während der Zeit unmittelbar vor ihrem Eintritt. Denn die Nachricht, nun einen Platz in der Klinik erhalten zu haben, scheint sie als deutlichen Einschnitt ihres Alltags zu erfahren, den sie zunächst nicht zuzuordnen weiß. Es wird deutlich, dass sie die Zeit zwischen der Anmeldung in der Klinik und dem Zeitpunkt, zu dem ein Platz frei wird, nicht oder nur sehr begrenzt mit Warten verbracht hat. Vielmehr bricht das Ereignis der von der Mutter überbrachten Mitteilung, dass sie einen Platz in der Jugendpsychiatrie erhalten hat, in die regulä- ren adoleszenten und von Sorglosigkeit motivierten Alltagsgestaltungen ein. Gleichzeitig zeigt die Normalität und Altäglichkeit Olivias Handelns - sie geht zur Schule und hat Freund*innen, die sie zu sich nach Hause einlädt - wie sehr die Krise während der langen Zeit des Wartens, die aufgrund der geringen Anzahl an Behandlungsplätzen nicht unüblich ist, in den Hintergrund getreten ist. Im Unterschied zu Sebastian, dessen Klinikaufenthalt aus einem langen Prozess der Krise schließlich in eine Eskalation mündet, erscheint der Klinikaufenthalt für Olivia als eine Handlungsoption unter anderen. Dadurch entsteht für sie gleichzeitig die Möglichkeit, die Entscheidung für einen Klinikaufenthalt für sich zu begründen und sich diese als Teil ihrer autonom gestalteten Lebensgeschichte anzueignen. Der Hintergrund der konkreten Entscheidungskrise, in der Olivia sich selbstermächtigend für den Klinikaufenthalt entscheidet, ist eine Erklärung für ihre Fähigkeit sich den Raum der Klinik anzueignen und therapeutisch fruchtbar zu machen. Diese Aneignung wird vor allem in Olivias Aussage im zweiten Einzelgespräch nach dem Klinikaufenthalt deutlich: „Olivia: [...] un weil früher hab ich so gedacht m: ich kann d-dies nich und ich bin sehr einseitig u:nd heute: ich hab ganz viele Möglichkeiten (.) und die Klinik hat mir wirklich sehr geholfen." Eben diese Offenheit gegenüber dem offerierten Angebot und die Anerkennung der Klinik als Autorität und Expertin in Bezug auf ihre eigene psychosoziale Krise zeigt sich auch in der Aussage Olivias Mutter im Anschluss an den Klinikaufenthalt ihrer Tochter: „Mutter: [...] so viel Elan den sie da aus der Klinik auch mitgebracht hat ne ich war ganz ganz platt eigentlich."

Mit dem Bündnis zwischen Olivia und der Klinik wird die Mutter-Tochter-Beziehung dahingehend unter Druck gesetzt, dass beide, um die Beziehung zu restabilisieren, die von der Tochter anerkannte Deutung der Klinik in ihren Beziehungsentwurf integrieren. In der gemeinsamen Aneignung der Krisendefinition wird der institutionelle Dritte in seiner versöhnenden Kraft in den Blick gerückt und damit verbundene Ambivalenzen bearbeitet. Die zuvor ebenfalls vorhandenen, aber deutlich weniger gravierenden Autonomiekonflikte zwischen Olivia und ihrer Mutter werden durch den institutionellen Dritten nicht - wie 
in Sebastians Fall - noch verschärft, sondern tragen tendenziell eher zur Harmonisierung bei. Die Perspektive der Klinik wird von Olivia und von ihrer Mutter nicht als gravierende Bedrohung familialer Verbindlichkeiten erfahren. Für Olivia steht der Klinikaufenthalt vielmehr im Zeichen ihrer Autonomie, da sie sich selbst für diesen entscheidet und dies für sich zu begründen weiß. Wenngleich sich bei Olivias Mutter eine größere Ambivalenz gegenüber der Deutung der Klinik im Vergleich zu Olivia zeigt, bedroht die Klinik das familiale Gefüge schon deswegen nicht, weil die Position der Klinik nicht weiter manifest, sondern nur als innere Bezugsgröße - vor allem für Olivia - fortbesteht. Ein wesentlicher Unterschied zum Fall Sebastian ist dabei auch, dass Olivias Klinikaufenthalt nicht als Erziehungsschwierigkeit gerahmt und demnach auch nicht als (Erziehungs-)Krise von Olivias Mutter erfahren wird.

\subsection{Fallkontrastiver Vergleich}

Es stellt sich in beiden Fällen die Frage, wie die Familie die Spannungen, die aus dem Übertrag von (Erziehungs-)Verantwortlichkeiten an die Jugendpsychiatrie entstehen, bearbeitet. Der Ausschluss des Kindes durch eine Koalition zwischen Eltern und Klinik und die machtvolle Infragestellung und Zurückweisung der adoleszenten Autonomie wirft in Sebastians Fall die Familie vor zentrale Fragen der Selbstvergewisserung. Die Koalition zwischen Eltern und Klinik, die durch die erzwungene Einweisung und die damit verbundene Infragestellung Sebastians Autonomie zutage tritt, ist zwar in den Interviews ein nicht verschweigbares Thema, sie wird aber trotzdem weitestgehend tabuisiert. Zentraler Fokus des Familiengesprächs ist die gemeinsame Ablehnung der Klinik nach Sebastians Entlassung. Nach dem Ausschluss Sebastians durch seine Eltern wird die Klinik zum Gegenspieler; sie ist es nun, die ihn loswerden möchte. Damit erfährt die Rolle der Eltern eine Umdeutung, wenn diese sich selbst zu „Retter*innen“ stilisieren, die dem heimatlosen Sohn wieder einen Platz in ihrer Mitte geben und damit die aus der Zwangseinweisung resultierenden Konflikte zu heilen versuchen. Während Sebastian sich als Spielball elterlicher und psychiatrischer Machtinstrumente erlebt, begreift Olivia ihre Erfahrung in der Klinik als lebensbiografische Weichenstellung, die ihr das Aufbrechen der eher symbiotischen Beziehung zur Mutter zu erleichtern scheint und es ihr dadurch ermöglicht, Entscheidungsräume, unabhängig von ihrem primärsozialisatorischen Milieu, zu erschließen. Während Sebastians Familie die Klinik ablehnt, um sich nach dem stationären Aufenthalt wieder als Familie zu stabilisieren, können Olivia und ihre Mutter sich die Perspektive der Klinik zu eigen machen, weil sie keine so elementare Bedrohung des familialen Gefüges darstellt. Es zeigt sich, dass dem institutionellen Dritten zwar durchaus eine machtvolle Position zukommt, die Dyadenbildung mit diesem allerdings durch seine zeitliche Begrenzung erschwert wird. Damit steht dieser auch nur temporär als manifeste Position im triadischen Gefüge mit den Eltern und Adoleszenten/Patient*innen zur Verfügung und ist von der inneren Aneignung der beiden anderen Parteien abhängig.

\section{ZUR RELEVANZ TRIADISCHER STRUKTU- REN IM KONTEXT SOZIALWISSENSCHAFTLI- CHER SOZIALISATIONSFORSCHUNG}

Der gezielte Blick auf triadisch konstellierte Beziehungsdynamiken - wie die mit einem jugendpsychiatrischen Klinikaufenthalt verbundenen Dreieckskonstellationen bestehend aus Familie bzw. Eltern, Adoleszenten und Klinik - ermöglicht es, die in Familien durch einen institutionellen Dritten angestoßenen Dynamiken zu fokussieren. Dabei haben wir gezeigt, dass die Berücksichtigung der spezifischen, mit der Lebensphase der Adoleszenz verbundenen Bedingungen eine wichtige Komponente darstellt, um zu verstehen, in welchen Spannungsfeldern sich die Beziehungsdynamiken mit dem institutionellen Dritten vollziehen.

Abgesehen von der mitunter invasiv auftretenden Deutungsmacht der Jugendpsychiatrie kann ihr Hinzutritt für die Familie die Möglichkeit zur Dezentrierung und Perspektivenerweiterung bergen. Dabei muss vor dem Hintergrund einer auf triadische Dynamiken ausgerichteten sozialisationstheoretischen Perspektive berücksichtigt werden, welche Bedeutung der Jugendpsychiatrie von den Adoleszenten und Familien zugeschrieben wird, welcher Art die zu behandelnde „Krankheit" ist und ob und wer einen Leidensdruck empfindet. Konkreter formuliert: Wessen 
Entscheidung ist es, eine Behandlung in einer Jugendpsychiatrie zu initiieren? Wird sie einstimmig von der Familie aufgesucht oder bilden einzelne Akteur*innen in der Konstellation des institutionellen Dreiecks eine Koalition, sodass ein Dritter ausgeschlossen wird? Wie wird der Klinikeintritt begründet und gibt es ein familial geteiltes Narrativ oder erzählen die Adoleszenten ihre eigene Geschichte? Welche Koalitionen entstehen innerfamilial, die dazu beitragen eine Behandlung zu erschweren, oder sie überhaupt erst zu ermöglichen? Und was bedeutet es für potenzielle Individuations- und Autonomiebestrebungen, wenn diese seitens der Eltern (und staatlicher Entscheidungsgewalt) durch eine Zwangseinweisung in Frage gestellt werden? Es ist vor dem Hintergrund des Hinzutritts des institutionellen Dritten daher nach den spezifischen Prozessen der (Neu-)Positionierung und der ReAktualisierung von Konflikten zu fragen, die der Dritte im familialen Gefüge zu dynamisieren in der Lage ist. Erst über die vielfältig sich formierenden adoleszenten Transformationsimpulse, die sowohl Transformations- und Ablösungswünsche, als auch imperative, Erwartungen und Anforderungen implizieren können, sind Aussagen über adoleszente Individuations- und familiale Bildungsprozesse möglich. Letztlich sensibilisiert die triadische Perspektive für den spezifischen Resonanzraum innerhalb der Familie, die eine Übernahme und Aneignung des Dritten - als sowohl strukturell dezentrierende Figur wie auch als solche, die spezifische, historisch gewachsene Deutungsangebote zur Verfügung stellt - ermöglicht oder verhindert. Hierdurch könnte sich der triadisch sensibilisierte Blick auch für die Betrachtung anderer Institutionen eignen, in denen sich das Spannungsfeld von Öffentlichkeit und Privatheit verschiebt.

Mit der Jugendpsychiatrie als Institution des medizinisch-therapeutischen Handlungssystems betrachten wir einen institutionellen Dritten, dessen dezentrierende und krisengenerierende und -bearbeitende Bedeutung sowohl über dessen fachliche Ausrichtung (Psychiatrie/Psychotherapie), als auch strukturelle Wirkmächtigkeit als "Dritter" zum Tragen kommt. Unsere bisherigen Erkenntnisse zeigen, dass sich ein psychiatrischer Aufenthalt für den Individuierungsprozess der Adoleszenten sowohl dynamisierend - als „potenzielle Quelle adoleszenter Neuschöpfung“
(Becher/Schulmeister 2020) - als auch hemmend auswirken kann. Über die Erweiterung bestehender familialer Beziehungskonstellationen und durch die Klinik dargebotene Deutungsangebote lassen sich unterschiedliche Modi familialer Krisenbearbeitung empirisch erschließen. In den vorgestellten Fällen tritt die Klinik in zwei differente Familienkonstellationen ein und dynamisiert diese auf unterschiedliche Weise: Im Fall Sebastian gerät sie als Bündnispartner der Eltern durch die erzwungene Einweisung als den adoleszenten Sohn deautonomisierendes Mittel der Erziehung in den Blick. Bei Olivia hingegen wird sie zum Bündnispartner für adoleszente Ablösungsprozesse, da diese sich eigenverantwortlich für eine stationäre Behandlung entscheidet und die mit der Behandlung verbundenen Deutungsangebote, unterstützt durch ihre Mutter, anzunehmen bereit ist.

Die hier ins Zentrum gerückte triadische Konstellation lässt sich hinsichtlich ihrer sozialisatorischen und biografieorganisierenden Wirkmächtigkeit erst vor dem Hintergrund der familialen Binnendynamiken und Krisendeutungen sowie den in diesem Dreieck wirksam werdenden Transformationsanforderungen begreifen. Mit der hier vorgeschlagenen heuristischen Perspektive zur Bedeutsamkeit der Figur des institutionellen Dritten plädieren wir dafür, die Arbeit an triadischen Konstellationen in intersubjektiven Aushandlungsprozessen als relevante Analysedimension in der Forschung zu Sozialisation in (geschlossenen) Institutionen, wie sie bei Neuber und Zahradnik (2019) unter Betonung unterschiedlicher struktureller Wechselbeziehungen vielfältig in den Blick geraten, zu berücksichtigen. Dies erfordert jedoch nicht nur die Rekonstruktion struktureller Krisenkonstellationen und damit verbundener Aushandlungsmodi. Vielmehr sind gerade vor dem Hintergrund der Lebensphase Adoleszenz in einem umfassenderen Zugriff Ermöglichungsbedingungen wie Begrenzungen für eigensinnige Positionen und Bildungs- und Autonomisierungsprozesse im Kontext der Lebenslage Krankheit/Gesundheit freizulegen. 


\section{LITERATUR}

Allert, Tilman (1998): Die Familie. Fallstudien zur Unverwüstlichkeit einer Lebensform. Berlin: Walter de Gruyter.

Becher, Julia/Schulmeister, Janina (2020): „Ich hab ganz viele Möglichkeiten und die Klinik hat mir wirklich sehr geholfen" - Die Kinder- und Jugendpsychiatrie als potenzielle Quelle adoleszenter Neuschöpfung. In: Thiersch, Sven (Hrsg.): Qualitative Längsschnittforschung - Bestimmungen, Forschungspraxis und Reflexionen. Opladen: Budrich, 249-271.

Bollig, Sabine/Kelle, Helga/Seehaus, Rhea (2012): (Erziehungs-)Objekte beim Kinderarzt. Zur Materialität von Erziehung in Kindervorsorgeuntersuchungen. In: Priem, Karin/König, Gudrun M./Casale, Rita (Hrsg.): Die Materialität der Erziehung: Kulturelle und soziale Aspekte pädagogischer Objekte. 58. Beiheft der Zeitschrift für Pädagogik. Weinheim: Beltz, 218-237.

Buchholz, Michael B. (1993): Dreiecksgeschichten. Eine klinische Theorie psychoanalytischer Familientherapie. Göttingen: Vandenhoeck \& Ruprecht.

Bütow, Birgit/Pomey, Marion/Rutschmann, Myriam/Schär, Clarissa/Studer, Tobias (2014) (Hrsg.): Sozialpädagogik zwischen Staat und Familie. Alte und neue Politiken des Eingreifens. Wiesbaden: VS.

Eßlinger, Eva/Schlechtriemen, Tobias/Schweitzer, Doris/Zons, Alexander (Hrsg.) (2010): Die Figur des Dritten. Ein kulturwissenschaftliches Paradigma. Frankfurt a. M.: Suhrkamp.

Fischer, Joachim (2008): Tertiarität. Die Sozialtheorie des „Dritten“ als Grundlegung der Kulturund Sozialwissenschaften. In: Raab, Jürgen/Pfadenhauer, Michaela/Stegmaier, Peter/Dreher, Jochen/Schnettler, Bernt (Hrsg.): Phänomenologie und Soziologie. Theoretische Positionen, aktuelle Problemfelder und empirische Umsetzungen. Wiesbaden: VS, 121-130.

Fivaz-Depeursinge, Elisabeth/Corboz-Warnery, Antoinette (2001): Das primäre Dreieck. Vater, Mutter und Kind aus entwicklungstheoretischsystemischer Sicht. Heidelberg: Carl-Auer.
Gehres, Walter/Hildenbrand, Bruno (2008): Identitätsbildung und Lebensverläufe bei Pflegekindern. Wiesbaden: VS.

Goffman, Erving (2014): Asyle. Über die soziale Situation psychiatrischer Patienten und anderer Insassen. 19. Auflage. Frankfurt a. M.: Suhrkamp.

Grieser, Jürgen (2017): Triangulierung. 2. Auflage. Gießen: Psychosozial-Verlag.

Haubl, Rolf/Liebsch, Katharina (Hrsg.) (2010): Mit Ritalin Leben. ADHS-Kindern eine Stimme geben. Göttingen: Vandenhoeck \& Ruprecht.

Hornung, Janina/Becher, Julia (2019): Von Förderschule zu Förderschule - Zum Motiv der Entlastung in der Rekonstruktion eines Übergabegesprächs nach dem Aufenthalt in einer Kinder- und Jugendpsychiatrie. In: Ellinger, Stephan/Schott-Leser, Hannah (Hrsg.): Rekonstruktionen sonderpädagogischer Praxis. Eine Fallsammlung für die Lehrerbildung. Opladen: Budrich, 75-102.

Janssen, Angela (2018): Verletzbare Subjekte. Grundlagentheoretische Überlegungen zur conditio humana. Opladen: Verlag Barbara Budrich.

King, Vera (2018): Die äußere und innere Bedeutung der Triade. Eine Rekonzeptualisierung angesichts pluralisierter Lebensformen. In: WestEnd - Neue Zeitschrift für Sozialforschung 15 (2), 87-103.

King, Vera (2015): Kindliche Angewiesenheit und elterliche Generativität. Subjekt- und kulturtheoretische Perspektiven. In: Andresen, Sabine/Koch, Claus/König, Julia (Hrsg.): Vulnerable Kinder. Interdisziplinäre Annäherungen. Wiesbaden: VS, 23-43.

King, Vera (2013): Die Entstehung des Neuen in der Adoleszenz. Individuation, Generativität und Geschlecht in modernisierten Gesellschaften. 2. Auflage. Wiesbaden: VS.

Koschorke, Albrecht (2010): Institutionentheorie. In: Eßlinger, Eva/Schlechtriemen, Tobias/Schweitzer, Doris/Zons, Alexander (Hrsg.): Die Figur des Dritten. Ein kulturwissenschaftliches Paradigma. Berlin: Suhrkamp, 4964.

Liebsch, Katharina/Haubl, Rolf/Brade, Josephin/Jentsch, Sebastian (2013): Normalität und Normalisierung von $A D(H) S$. Prozesse und 
Mechanismen der Entgrenzung von Erziehung und Medizin. In: Kelle, Helga/Mierendorff, Johanna (Hrsg.): Normierung und Normalisierung der Kindheit. Weinheim: Beltz, 158-175.

Neuber, Anke/Zahradnik, Franz (Hrsg.) (2019): Geschlossene Institutionen - Theoretische und empirische Einsichten. Weinheim: Beltz.

Oevermann, Ulrich (2004): Sozialisation als Prozess der Krisenbewältigung. In: Geulen, Dieter (Hrsg.): Sozialisationstheorie Interdisziplinär. Stuttgart: Lucius \& Lucius, 155-182.

Oevermann, Ulrich (2001): Die Soziologie der Generationenbeziehungen und der historischen Generationen aus strukturalistischer Sicht und ihre Bedeutung für die Schulpädagogik. In: Kramer, Rolf-Torsten/Helsper, Werner/Busse, Susann (Hrsg.): Pädagogische Generationsbeziehungen. Jugendliche im Spannungsfeld von Schule und Familie. Opladen: Leske + Budrich, 78-128.

Oevermann, Ulrich (2000): Die Methode der Fallrekonstruktion in der Grundlagenforschung sowie der klinischen und pädagogischen Praxis. In: Kraimer, Klaus (Hrsg.): Die Fallrekonstruktion. Sinnverstehen in der sozialwissenschaftlichen Forschung. Frankfurt a. M.: Suhrkamp, 58-156.

Oevermann, Ulrich (1996): Theoretische Skizze einer revidierten Theorie professionalisierten Handelns. In: Combe, Arno/Helsper, Werner (Hrsg.): Pädagogische Professionalität. Frankfurt a. M.: Suhrkamp, 70-183.

Peter, Claudia (2006): Beziehungskonstellationen zwischen Patienten und Professionellen im organisatorischen Kontext des Krankenhauses: drei Beispiele. In: Zeitschrift für qualitative Bildungs-, Beratungs- und Sozialforschung, 7 (1), 69-92.

Rüth, Ulrich (2011): Die Unterbringung in der Kinder- und Jugendpsychiatrie. In: Familie Partnerschaft Recht 12, 554-557.

Silkenbeumer, Mirja/Becher, Julia/Schulmeister, Janina (2020): Patient werden und Schüler blei- ben. Fallstudie zur bildungsbiografischen Bearbeitung von Übergängen im Schnittfeld von Familie, Jugendpsychiatrie und (Klinik-)Schule. In: Thiersch, Sven/Silkenbeumer, Mirja/Labede, Julia (Hrsg.): Individualisierte Übergänge. Aufstiege, Abstiege und Umstiege im Bildungssystem. Wiesbaden: VS, 151-184.

Simmel, Georg (1992): Die quantitative Bestimmtheit der Gruppe. In: Rammstedt, Otthein (Hrsg.): Soziologie: Untersuchungen über die Formen der Vergesellschaftung. Frankfurt a. M.: Suhrkamp, 63-159.

Stierlin, Helm (2001): Psychoanalyse - Familientherapie-systemische Therapie. Entwicklungslinien, Schnittstellen, Unterschiede. Stuttgart: Klett-Cotta.

Stierlin, Helm/Levi, David/Savard, Robert (1980): Zentrifugale und zentripetale Ablösung in der Adoleszenz: zwei Modi und einige ihrer Implikationen. In: Döbert, Rainer/Habermas, Jürgen/Nummer-Winkler, Gertrud (Hrsg.): Entwicklung des Ichs. Königstein: Anton Hain Meisenheim, 46-67.

Thiersch, Sven (2020): Qualitative Längsschnittforschung. Eine Einleitung. In: Thiersch, Sven (Hrsg.): Qualitative Längsschnittforschung. Bestimmungen, Forschungspraxis und Reflexionen. Opladen: Budrich, 9-27.

von Freyberg, Thomas/Wolff, Angelika (Hrsg.) (2005): Störer und Gestörte. Band 1: Konfliktgeschichten nicht beschulbarer Jugendlicher. Frankfurt a.M.: Brandes \& Apsel.

Wernet, Andreas (2020): Identitätskrise, Transformation und Ausdrucksgestalt: Zum rekonstruktionsmethodologischen Problem des "Gewordenseins". In: Thiersch, Sven (Hrsg.): Qualitative Längsschnittforschung. Bestimmungen, Forschungspraxis und Reflexionen. Opladen: Verlag Barbara Budrich, 127-145.

Wernet, Andreas (2009): Einführung in die Interpretationstechnik der objektiven Hermeneutik. Wiesbaden: VS. 


\section{Zu den Autor*innen}

Julia Becher ist wissenschaftliche Mitarbeiterin am Fachbereich Erziehungswissenschaften der GoetheUniversität Frankfurt am Main. Ihre Forschungsschwerpunkte liegen in den Bereichen der Familien-, Adoleszenz- und Sozialisationsforschung.

Janina Schulmeister ist wissenschaftliche Mitarbeiterin am Fachbereich Erziehungswissenschaften der Goethe-Universität Frankfurt am Main. Ihre Forschungsschwerpunkte liegen in den Bereichen der Familien-, Adoleszenz- und Sozialisationsforschung.

Mirja Silkenbeumer ist Professorin für Erziehungswissenschaft am Institut für Sonderpädagogik der Goethe-Universität Frankfurt am Main. Ihre Arbeits- und Forschungsschwerpunkte liegen in den Bereichen der Adoleszenz- und Familienforschung, Forschung zu Übergängen in Biografie und Lebenslauf, rekonstruktive Professionalisierungs- und Bildungsforschung und dem Bereich pädagogischer Beratung.

\section{Kontakt}

Julia Becher

Goethe-Universität Frankfurt am Main

Fachbereich Erziehungswissenschaften

Institut für Sonderpädagogik

Theodor-W.-Adorno-Platz 6

D-06110 Frankfurt am Main

E-Mail: becher@em.uni-frankfurt.de

URL: https://www.uni-frankfurt.de/55945745/Julia_Becher_M_A

Janina Schulmeister

Goethe-Universität Frankfurt am Main

Fachbereich Erziehungswissenschaften

Institut für Sonderpädagogik

Theodor-W.-Adorno-Platz 6

D-06110 Frankfurt am Main

E-Mail: j.schulmeister@em.uni-frankfurt.de

URL: https://www.uni-frankfurt.de/55945808/Janina_Schulmeister_M_A

Prof. Dr. Mirja Silkenbeumer

Goethe-Universität Frankfurt am Main

Fachbereich Erziehungswissenschaften

Institut für Sonderpädagogik

Theodor-W.-Adorno-Platz 6

D-06110 Frankfurt am Main

E-Mail: silkenbeumer@em.uni-frankfurt.de

URL: : https://www.uni-frankfurt.de/55945511/Prof_Dr_Mirja_Silkenbeumer 